\title{
ilmedia
}

Zentner, Lena :

Mathematical synthesis of compliant mechanism as cochlear implant

Publikation entstand im Rahmen der Veranstaltung:

The first Workshop on Microactuators and Micromechanisms :

MAMM 2010 ; Aachen, Germany, May 27-29, 2010, 4 S. 


\title{
The First Workshop on Microactuators and Micromechanisms \\ MAMM 2010
}

\section{MATHEMATICAL SYNTHESIS OF COMPLIANT MECHANISM AS COCHLEAR IMPLANT}

\author{
L. Zentner ${ }^{1}$
}

\begin{abstract}
Cochlear implants can be successfully used to reduce the inner ear profound deafness. The implant is inserted into the inner ear by the surgeon's hand. Because the cochlea has a spiral-like structure (cochlear duct), the insertion of the implant is often difficult, furthermore the basilar membrane could be easily damaged. One of the aims of our investigation is to develop a mathematical model based an synthesis method for implants with hydraulic actuation. This hydraulic actuation, which is integrated in the implant, facilitates the insertion of the implant structure to the shape of cochlear duct. Thus, the implant can follow the spiral-shaped cochlear duct without damaging the sensitive tissue of the basilar membrane. Some examples for hydraulic actuated cochlear implants based on compliant mechanisms technology are presented in this paper.
\end{abstract}

Key words: compliant mechanism, cochlea implant, hydraulic device, mathematical modelling

\section{Introduction}

Cochlear-implants are implanted for patients with profound deafness. The people, who have implantation directly after hearing loss, are still able to communicate. Early treated children develop their language competence and speaking reproduction in such way that a great number of them are able to go to normal school. Since the first beginning of implantation 45 years ago, cochlear-implants have been developed further intensively. All of the nowadays applied implants, which are implanted in the scala tympani (Fig. 1), consist of an electrode arrey, a receiver and a stimulator unit for signal, an electrical transmission and a receiving antenna. In the Fig. 3a is a schematic representation of an implant from MED-EL (MED-EL, Innsbruck, Austria). 12 platinum electrode pairs are embedded in one soft silicone-carrier. The cochlear hair cells and auditory nerve fibres are located on the inner side of scala tympani, from above the scala tympani is terminated with a thin sensitive basilar membrane of the remaining part of cochlear duct (Fig. 1). By insertion of the implant into the cochlea the tissue can be damaged, which can cause destruction of reminder hearing. The preformed implants can also cause undesirable injuries [1]. An implant, which can deform itself during the operation and are able to suit bit by bit to the shape of cochlear duct, can simplify the insertion procedure and can so prevent the insertion trauma of cochlea. This deformation can be achieved via a design modification of a compliant mechanism [4], [5].

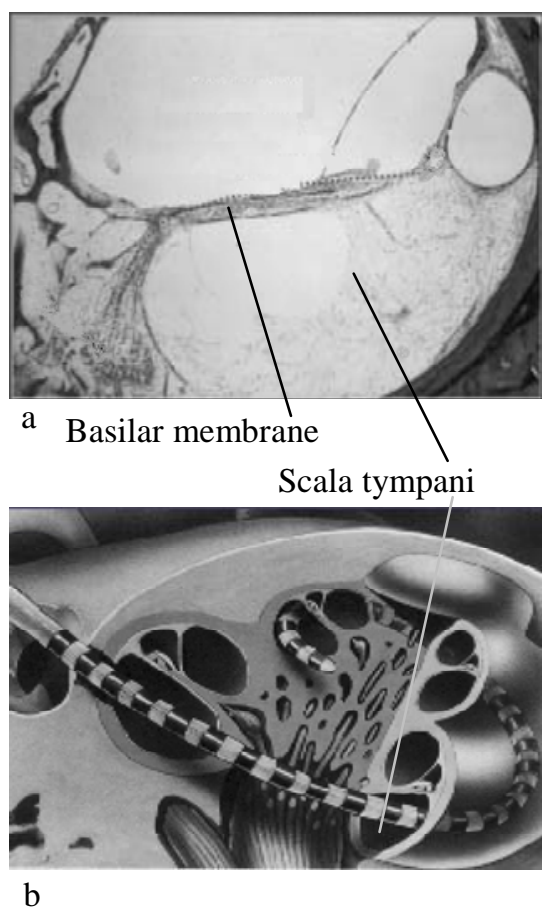

Fig. 1. $a$-Cochlear duct; $b$ - Cochlea

There are two ways to realize the required

\footnotetext{
${ }^{1}$ Technische Universität Ilmenau, Germany.
} 
deformation:

1. concentrically hollow in the silicone carrier with geometric asymmetry. The geometric asymmetry can be realized by embedding of materials with different mechanical properties in the silicone carrier or by a special arrangement of the electrode wires in silicone carrier

2. non-concentrically arrangement of the hollow in the silicone carrier [6].

Further the form of the implant with an embedded fibre which corresponds to the form of the cochlea by pressure in the hollow is searched.

\section{Representation of the cochlea centre line as a space curve}

The form of the centre line of the cochlea duct is shown in the Fig. 2 in the cylindrical coordinate system with coordinates $\mathrm{r}$ and $\mathrm{z}$ which are functions of the angle $\varphi[2]$ :

$$
\begin{aligned}
& \mathrm{r}=\frac{\mathrm{k}_{1}\left(\mathrm{k}_{2}+\pi\right)}{\left(2 \mathrm{k}_{2}+\pi\right)\left(\mathrm{k}_{2}+\varphi\right)}, \\
& \mathrm{z}=\mathrm{k}_{3}\left(\mathrm{e}^{\mathrm{k}_{4} \varphi}-1\right) .
\end{aligned}
$$

The constants in these equations are

$$
\mathrm{k}_{1}=10, \mathrm{k}_{2}=1, \mathrm{k}_{3}=0.8, \mathrm{k}_{4}=0.1 \text {. }
$$

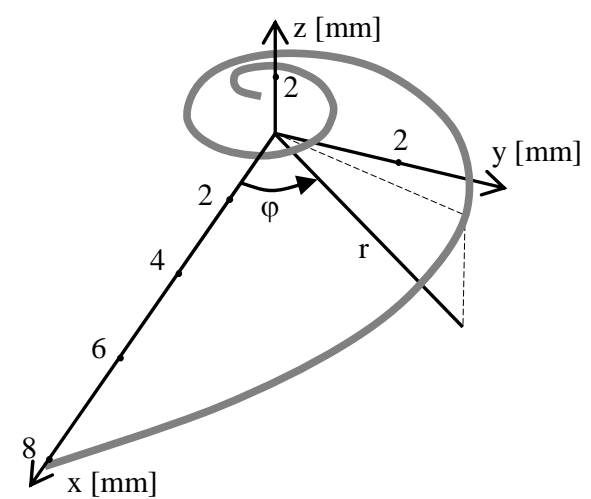

Fig. 2.: Form of the centre line of the cochlear duct

The length of the curve element of the cochlea centre line is

$$
\mathrm{ds}=\sqrt{\mathrm{dr}^{2}+\mathrm{dz}^{2}} \text {. }
$$

Division by d $\varphi$ yields

$$
\frac{\mathrm{ds}}{\mathrm{d} \varphi}=\sqrt{\left(\frac{\mathrm{dr}}{\mathrm{d} \varphi}\right)^{2}+\left(\frac{\mathrm{dz}}{\mathrm{d} \varphi}\right)^{2}} .
$$

Associated boundary condition is $s(0)=0$. This is a non-linear equation in terms of $s(\varphi)$. It can be solved only numerically. For further calculations $\varphi(\mathrm{s})$ is also necessary. Now the curvature $\kappa_{3}$ and the torsion $\kappa_{1}$ of the cochlea centre line can be expressed by derivatives of Cartesian coordinates with respect to $\varphi$ :

$$
\begin{array}{r}
\kappa_{3}^{2}(\varphi)=\frac{\left(x^{\prime 2}+y^{\prime 2}+z^{\prime 2}\right)\left(x^{\prime \prime 2}+y^{\prime \prime 2}+z^{\prime 2}\right)}{\left(x^{\prime 2}+y^{\prime 2}+z^{\prime 2}\right)^{3}} \\
-\frac{\left(x^{\prime} x^{\prime \prime}+y^{\prime} y^{\prime \prime}+z^{\prime} z^{\prime \prime}\right)^{2}}{\left(x^{\prime 2}+y^{\prime 2}+z^{\prime 2}\right)^{3}},
\end{array}
$$

$$
\kappa_{1}(\varphi)=\frac{\left|\begin{array}{ccc}
x^{\prime} & y^{\prime} & z^{\prime} \\
x^{\prime \prime} & y^{\prime \prime} & z^{\prime \prime} \\
x^{\prime \prime \prime} & y^{\prime \prime \prime} & z^{\prime \prime \prime}
\end{array}\right|}{\kappa_{3}^{2}\left(x^{\prime 2}+y^{\prime 2}+z^{\prime 2}\right)^{3}} .
$$

By using of the numerically calculated term $\varphi(s)$ the curvature and torsion of the centre line can be expressed dependent on $\mathrm{s}$. The parameters $\kappa_{1}(\mathrm{~s})$ and $\kappa_{3}(\mathrm{~s})$ are well suitable to describe the form of the cochlea duct.

\section{Modelling of the implant}

An insertion implant from MED EL Innsbruck is shown in Fig. 3a. In Fig. 3b-c the structure is shown, which is modelled. The radius of the implant changes from $r_{a}=0.65 \mathrm{~mm}$ to $r_{e}=0.25 \mathrm{~mm}$. The function $r(s)$ can be expressed by second-order polynomial:

$$
r(s)=\frac{r_{a}-r_{e}}{l^{2}}(s-l)^{2}+r_{e}
$$

Geometrical moments of inertia are expressed by equations (9).

$$
\mathrm{I}_{1}=2 \mathrm{I}_{2}=2 \mathrm{I}_{3}=\frac{\pi}{2}\left(\left(\frac{\mathrm{r}_{\mathrm{a}}-\mathrm{r}_{\mathrm{e}}}{\mathrm{l}^{2}}(\mathrm{~s}-\mathrm{l})^{2}+\mathrm{r}_{\mathrm{e}}\right)^{4}-\mathrm{r}_{\mathrm{i}}^{4}\right) \text {. }
$$

The matrix A with E and G as Young's modulus and shear modulus is expressed as follows:

$$
\mathbf{A}=\left(\begin{array}{ccc}
\mathrm{GI}_{1} & 0 & 0 \\
0 & \mathrm{EI}_{2} & 0 \\
0 & 0 & \mathrm{EI}_{3}
\end{array}\right)
$$




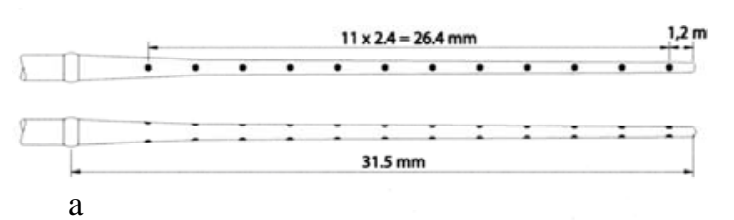

a

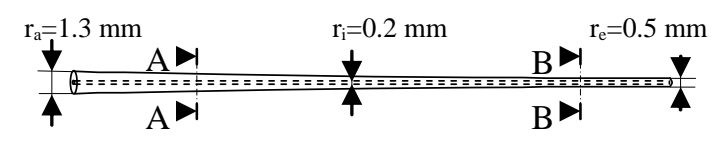

b

c 8 hollow

B-B

(O)

Fig. 3. $a-$ Schematic representation of an implant from MED EL Innsbruck; $b$-Geometric parameters of the implant model; $c$-Cross-section views of the implant model

In the implant model there is a hollow with constant radius $r_{i}$. In order to occur the bending of the implant, an unstretchable thin fibre of length $l$ is embedded in the wall with $\mathrm{h}$ distance from the symmetry axes of implant (Fig. 4). With inner pressure of fluid $\mathrm{p}$ in the hollow the implant structure will bend towards the embedded fibre.

The following model is set up, in order to find the form of the fibre for non-deformed shape of implant. The form of cochlea duct shall be reproduced by implant which is under pressure. The model is based on the theory of curved beams with hollows [3]. Linear material law is for this limited deformation supposed but large displacements are possible. The fibre corresponds to neutral fibre of the implant.
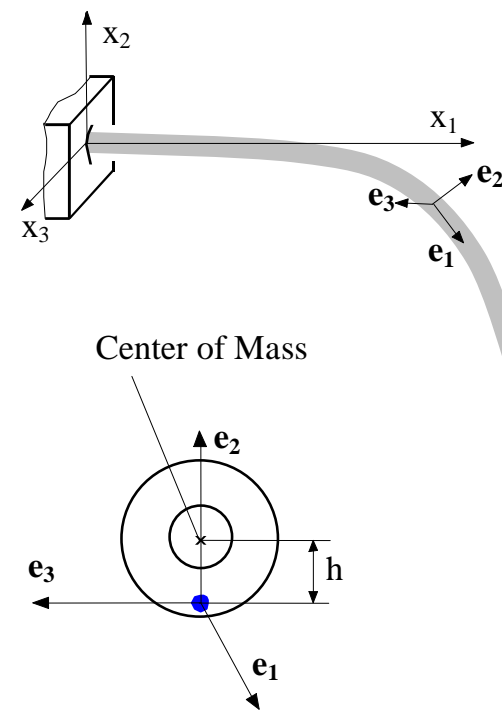

Fig. 4. Beam with embedded material and with round cross section

We introduce two orthogonal coordinate systems: fixed Cartesian coordinate system $\mathrm{x}_{\mathrm{j}}$ and moving one with unit vectors $\vec{e}_{j}$ (Fig. 4), attached to the neutral line. The distance between the neutral line (embedded material) and the centre of mass is h, which is small in comparison to the length and the radius of curvature of the implant. Therefore the parameter $s$ in equation (4) corresponds approximately to the length of an arch of the neutral line. It is measured from the fixed end to the free end. The following notations are used: $\overrightarrow{\mathrm{Q}}=\mathrm{Q}_{1} \overrightarrow{\mathrm{e}}_{1}+\mathrm{Q}_{2} \overrightarrow{\mathrm{e}}_{2}+\mathrm{Q}_{3} \overrightarrow{\mathrm{e}}_{3}$ as the vector of internal forces, with axial force $\mathrm{Q}_{1}$ and transverse forces $Q_{2}, \quad Q_{3} ; \quad \overrightarrow{\mathrm{M}}=\mathrm{M}_{1} \overrightarrow{\mathrm{e}}_{1}+\mathrm{M}_{2} \overrightarrow{\mathrm{e}}_{2}+\mathrm{M}_{3} \overrightarrow{\mathrm{e}}_{3}$ - vector of internal moments, where $\mathrm{M}_{1}$ - torsion moment, $\mathrm{M}_{2}, \mathrm{M}_{3}$ - bending moments; $\mathrm{P}_{0}=\mathrm{p} \pi r_{i}^{2}$ is force on the inner cross-section of a beam (implant).

The parameter $\vec{\kappa}$ is determined by the following relation:

$$
\frac{\mathrm{d} \overrightarrow{\mathrm{e}}_{\mathrm{i}}}{\mathrm{ds}}=\vec{\kappa} \times \overrightarrow{\mathrm{e}}_{\mathrm{i}}
$$

The initial form of the neutral fibre is given by $\kappa_{10}, \kappa_{20}$ and $\kappa_{30}$. The form of beam under pressure is described by following vector-equations:

$$
\begin{aligned}
& \frac{\mathrm{d} \overrightarrow{\mathrm{Q}}}{\mathrm{ds}}-\frac{\mathrm{d}\left(\mathrm{P}_{0} \overrightarrow{\mathrm{e}}_{1}\right)}{\mathrm{ds}}=\overrightarrow{0}, \\
& \frac{\mathrm{d} \overrightarrow{\mathrm{M}}}{\mathrm{ds}}+\left(\overrightarrow{\mathrm{e}}_{1} \times \overrightarrow{\mathrm{Q}}\right)=\overrightarrow{0}, \\
& \overrightarrow{\mathrm{M}}=\mathbf{A}\left(\vec{\kappa}-\vec{\kappa}_{0}\right) .
\end{aligned}
$$

The force equilibrium conditions for cochlea implant have following form:

$$
\begin{aligned}
& \frac{\mathrm{dQ}_{1}}{\mathrm{ds}}+\mathrm{Q}_{3} \kappa_{2}-\mathrm{Q}_{2} \kappa_{3}=0, \\
& \frac{\mathrm{dQ}}{\mathrm{ds}}+\left(\mathrm{Q}_{1}-\mathrm{P}_{0}\right) \kappa_{3}-\mathrm{Q}_{3} \kappa_{1}=0, \\
& \frac{\mathrm{dQ}_{3}}{\mathrm{ds}}+\mathrm{Q}_{2} \kappa_{1}-\left(\mathrm{Q}_{1}-\mathrm{P}_{0}\right) \kappa_{2}=0 .
\end{aligned}
$$

The corresponding boundary conditions are:

$$
\mathrm{Q}_{1}(\mathrm{l})=\mathrm{P}_{0}, \mathrm{Q}_{2}(\mathrm{l})=0, \mathrm{Q}_{3}(\mathrm{l})=0
$$

The moments can be expressed by $\kappa_{1}, \kappa_{2}$ and $\kappa_{3}:$ 


$$
\begin{aligned}
& \mathrm{EI}_{3}\left(\frac{\mathrm{d} \kappa_{3}}{\mathrm{ds}}-\frac{\mathrm{d} \kappa_{30}}{\mathrm{ds}}\right)+\mathrm{EI}_{2}\left(\kappa_{2}-\frac{\mathrm{d} \kappa_{20}}{\mathrm{ds}}\right) \kappa_{1}- \\
& \mathrm{GI}_{1}\left(\kappa_{1}-\kappa_{10}\right) \kappa_{2}+\mathrm{Q}_{2}=0, \\
& \mathrm{EI}_{2}\left(\frac{\mathrm{d} \kappa_{2}}{\mathrm{ds}}-\frac{\mathrm{d} \kappa_{20}}{\mathrm{ds}}\right)+\mathrm{GI}_{1}\left(\kappa_{1}-\frac{\mathrm{d} \kappa_{10}}{\mathrm{ds}}\right) \kappa_{3}- \\
& \mathrm{EI}_{3}\left(\kappa_{3}-\kappa_{30}\right) \kappa_{1}+\mathrm{Q}_{3}=0, \\
& \mathrm{GI}_{1}\left(\frac{\mathrm{d} \kappa_{1}}{\mathrm{ds}}-\frac{\mathrm{d} \kappa_{10}}{\mathrm{ds}}\right)+\mathrm{EI}_{3}\left(\kappa_{3}-\frac{\mathrm{d} \kappa_{30}}{\mathrm{ds}}\right) \kappa_{2}- \\
& \mathrm{EI}_{2}\left(\kappa_{2}-\kappa_{20}\right) \kappa_{3}=0 .
\end{aligned}
$$

The corresponding boundary conditions have the form:

$$
\begin{aligned}
& \kappa_{30}(l)=\kappa_{3}(l)-\frac{P_{0} h}{E_{3}(l)}, \\
& \kappa_{20}(l)=\kappa_{2}(l), \\
& \kappa_{10}(l)=\kappa_{1}(l) .
\end{aligned}
$$

The solution of these equations of a static problem is the unload shape of the implant for cochlea implant $\kappa_{10}(\mathrm{~s}), \kappa_{20}(\mathrm{~s})$ and $\kappa_{30}(\mathrm{~s})$. This shape of implant is deformed under given pressure to the end-form corresponding with the form of cochlea duct.

The Fig. 5 shows the form of fibre in the Cartesian coordinate system. The transformation formulas for $\kappa_{10}(\mathrm{~s}), \quad \kappa_{20}(\mathrm{~s}) \quad$ and $\quad \kappa_{30}(\mathrm{~s})$ into Cartesian coordinates are not presented here because of complexity of the expressions.

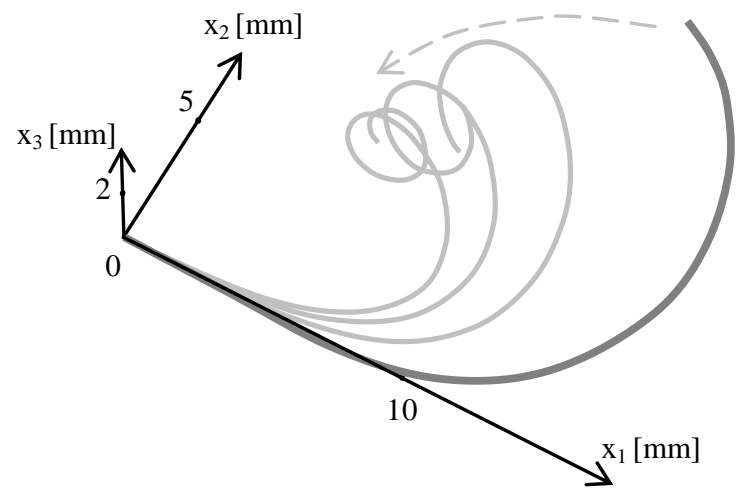

Fig. 5. Form of fibre in the Cartesian coordinate system

The calculations of the deformation of a threedimensional structure were carried out with the parameters $\mathrm{l}=31.5 \mathrm{~mm}, \mathrm{~h}=0.25 \mathrm{~mm}, \mathrm{E}=12.6 \mathrm{kN} / \mathrm{m}^{2}$ and the inner pressure $3 \mathrm{kN} / \mathrm{m}^{2}$.

\section{Summary}

In this paper it is shown that it is possible to realize an active bending of an implant. The theory of curved beams allows the description of deformation of the implant. It was shown, that the form of the implant which corresponds to the cochlea duct under defined pressure can be found.

As an alternative possibility thermo-gels are examined. By insertion the implant into the cochlear duct, temperature of gel increases to the value of the body temperature. Thereby the gel volume enlarges. The inner pressure in the hollow of the implant increases. As a result the implant is deformed and facilitates on this way the surgery.

\section{References}

1. Marangos, N, Laszig, R.: Cochlear Implants, HNO, Springer-Verlag, Physics and Mystery (1998) 46: pp. 12-26

2. Rau, T.; Eilers, H,; Leinung, M.; Hussong, A.; Lenarz, T.; Majdani, O.: Geometriemodellierung des Innenohres für die Cochlea Implantation - anatomische Variabilität und patientenspezifische Insertionsplanung ; 79. Jahresversammlung der Deutschen Gesellschaft für Hals-Nasen-OhrenHeilkunde, Kopf- und Hals-Chirurgie. Bonn, 30.04.-04.05.2008 Düsseldorf: German Medical Science GMS Publishing House; (2008)

3. Zentner, L.: Untersuchung und Entwicklung nachgiebiger Strukturen, Ilmenau ISLE Verlag, ISBN 3-932633-77-6, (2003)

4. Zentner, L.; Böhm, V.: On the Classification of Compliant Mechanisms. - In: Proceedings of the EUCOMES 08 / European Conference on Mechanism Science ; 2 (Cassino, Italy) : 2008.09.17-20. - Berlin : Springer (2009), pp. 431-438

5. Zentner, L.; Böhm, V.; Minchenya, V.: On the new reversal effect in monolithic compliant bending mechanisms with fluid driven actuators. - In: Mechanism and machine theory. - Amsterdam [u.a.]: Elsevier Science, Bd. 44 (2009), 5, pp. 1009-1018

6. Zentner, L.; Keskeny. J.; Westhofen, M.; Huba, A.: Hydraulic actuation for the navigation of a cochlear implant. - In: Actuator 2006 / International Conference on New Actuators; 10 (Bremen): 2006.06.14-16. - Bremen: HVG Hanseatische Veranst.-GmbH, Div. Messe Bremen (2006), pp. 980-984 